\title{
A TEORIA DA SOLUÇÃO INVENTIVA DE PROBLEMAS INTEGRADA À PRODUÇÃO MAIS LIMPA: ABORDAGEM TEÓRICA
}

\section{THE THEORY OF INVENTIVE PROBLEM SOLVING INTEGRATED TO THE CLEANER PRODUCTION: THEORETICAL APPROACH}

\author{
Flávio Issao Kubota* flavioissao.kubota@gmail.com \\ Leandro Cantorski da Rosa** leski78@hotmail.com \\ Universidade Federal de Santa Maria, Santa Maria, RS
}

\begin{abstract}
Resumo: Esforços visando à redução de impacto ambiental negativo por parte das empresas são um processo de resolução de problemas, os quais muitas vezes necessitam de soluções inventivas que gerem benefícios ambientais, técnicos e econômicos. Assim, esta pesquisa objetivou verificar, por meio da literatura, e discutir a integração da Teoria da Solução Inventiva de Problemas (TRIZ) às estratégias da produção mais limpa (PML), visando à estruturação de uma nova metodologia para a geração de soluções inventivas voltadas à redução de efluentes, resíduos e emissões. Para realizar este estudo, procedeu-se uma pesquisa de caráter qualitativo e natureza descritiva, com estratégia de coleta documental de dados. Os resultados evidenciam que há predominância de trabalhos no nível 1 da PML, com maior enfoque em melhorias no produto, uma quantidade menor de pesquisas em modificações no processo (nível 1) e em reciclagem interna (nível 2), e não se obteve evidências da TRIZ utilizada no nível 3 (reciclagem externa e ciclos biogênicos). A integração proposta é promissora, uma vez que ambas as metodologias buscam a situação ideal e a redução de problemas. Ao final, detectaram-se oportunidades para aplicação da TRIZ em processos produtivos, por meio da análise funcional e do Resultado Final Ideal (RFI), podendo essas ferramentas direcionar as equipes para a otimização de sistemas técnicos.
\end{abstract}

Palavras-chave: TRIZ. Produção mais limpa. Ecodesign. Melhoria de processos.

Abstract: Efforts towards to reduction of environmental impact by enterprises are a solving problem process, which often need inventive solutions that generate environmental, technical and economic benefits. Hence, this research aimed to verify, through literature, and discuss the Theory of Inventive Problem Solving (TRIZ) integration to cleaner production (CP) strategies, in order to structure a new methodology for inventive solutions focused on reduction of effluents, waste and emissions. To perform this study, we proceeded a qualitative and descriptive research, with documentary data collection strategy. The results show predominance of CP level 1 researches, with greater focus on product improvement, a smaller quantity of process modification studies (level 1 ) and internal recycling (level 2), and no evidence of TRIZ utilization was obtained on level 3 (external recycling and biogenic cycles). The proposed integration is promising, since both methodologies aim ideal situation and problems' reduction. At the end, opportunities were detected for application of TRIZ in production processes, by means of functional analysis and Ideal Final Result (IFR), being possible to direct teams towards optimization of technical systems.

Keywords: TRIZ. Cleaner production. Ecodesign. Process improvement. 


\section{INTRODUÇÃO}

A busca por soluções criativas dentro da indústria é um processo de resolução de problemas. Os esforços voltados para o estudo e implantação - ou incremento - de processos produtivos industriais são maneiras de resolver um problema durante o desenvolvimento do produto/processo/serviço em questão. Nesse contexto, Chehebe (1997) afirma que a natureza dos problemas ambientais é parcialmente atribuída à complexidade dos processos industriais utilizados pelo homem, pois todo produto, não importa de que material seja feito ou a finalidade de uso, provoca um impacto no meio ambiente, em função de seu processo produtivo, das matérias-primas que se consome ou devido ao seu uso ou disposição final.

Além disso, o direcionamento para melhores práticas ambientais é outro aspecto o qual vem sendo requisitado pelas partes interessadas aos negócios das organizações, uma vez que a maior parcela dos recursos utilizados nos processos não é renovável (KUBOTA; SILVA FILHO; ROSA, 2010). Desse modo, a produção mais limpa (PML) visa à redução de emissões e minimização no uso de energia, consideradas como funções indesejadas nos processos produtivos das empresas. Para a United Nations for Industrial Development Organization (UNIDO), a PML (em inglês cleaner production - CP) implica - para processos produtivos - na conservação de matéria-prima e energia e eliminação de materiais tóxicos, bem como a redução em quantidade e toxicidade de todas as emissões e desperdícios antes de deixarem o processo. A PML, conforme Rivera et al. (2009), é alcançada através de aplicações de conhecimento, aprimoramento de tecnologias e mudança de atitudes no trabalho. Essa última, mais conhecida como boas práticas.

Em paralelo, Zhang e Shang (2010) afirmam que a Teoria da Solução Inventiva de Problemas (TRIZ) é considerada um tipo de teoria inovadora, que funciona principalmente resolvendo contradições. Essa metodologia (conforme os mesmos autores), baseada na evolução de sistemas técnicos, é composta de vários tipos de métodos, cálculos para resolver problemas técnicos e exploração inovadora, de acordo com a compreensão global na resolução do sistema. Aplicações da TRIZ para os problemas relacionados a design de produtos para sustentabilidade cooperativa e eco-eficiência são documentadas na literatura, entretanto são raros (CHEN; LIU, 2001; KOBAYASHI, 2006). E o uso explícito da TRIZ dentro da PML 
não foi documentado até hoje, de acordo com o conhecimento dos autores (FRESNER et al., 2010). Além disso, Carvalho e Back (2001) e Zhang e Shang (2010) relatam que a TRIZ vem sendo aplicada nos mais diversos campos de conhecimento.

Baseando-se nesses fatos e informações, este artigo objetivou verificar, através da literatura, e discutir a aplicação da Teoria da Solução Inventiva de Problemas (TRIZ) como uma nova metodologia de estruturação da produção mais limpa (PML) nas organizações, visando à melhoria contínua com foco em soluções simples e inovadoras nessa área. Para isso, foram desenvolvidos os seguintes objetivos específicos: identificar a relação entre a TRIZ e a PML; identificar desvantagens e limitações, e; identificar pontos fortes e vantagens.

Justifica-se este estudo devido à possibilidade de maior compreensão da Teoria Inventiva da Solução de Problemas (TRIZ), a qual tem pouco material didático de aplicação em português e vem sendo crescentemente disseminada nas empresas de pequeno, médio e grande porte (CARVALHO; FERREIRA, 2005), bem como de sua aplicabilidade na PML. Os resultados e discussões acerca dessa pesquisa proporcionam fatores que facilitam e/ou limitam a aplicação da TRIZ como metodologia suporte à estratégia de produção mais limpa, de acordo com os autores deste artigo.

\section{FUNDAMENTAÇÃO TEÓRICA}

\subsection{Produção mais limpa - Definições}

O termo produção mais limpa (PML) possui diversas conotações (THRANE; NIELSEN; CHRISTENSEN, 2009). A PML é a aplicação contínua de uma estratégia ambiental preventiva e integrada para processos, produtos e serviços, visando aumentar a eficiência e reduzir riscos para os humanos e o meio ambiente (BERKEL, 2001; PIMENTA; GOUVINHAS, 2007; MEDEIROS et al., 2007; UNEP, 2006 apud THRANE; NIELSEN; CHRISTENSEN, 2009). A UNEP (2002), assim como Medeiros et al. (2007), distinguem seis tipos de soluções (Figura 1): boas práticas (housekeeping), reutilização e reciclagem, substituição de materiais e 
químicos perigosos, otimização de processos, mudança tecnológica e inovações e desenvolvimento de produtos mais limpos (ecodesign).

Figura 1 - Níveis de atuação da produção mais limpa

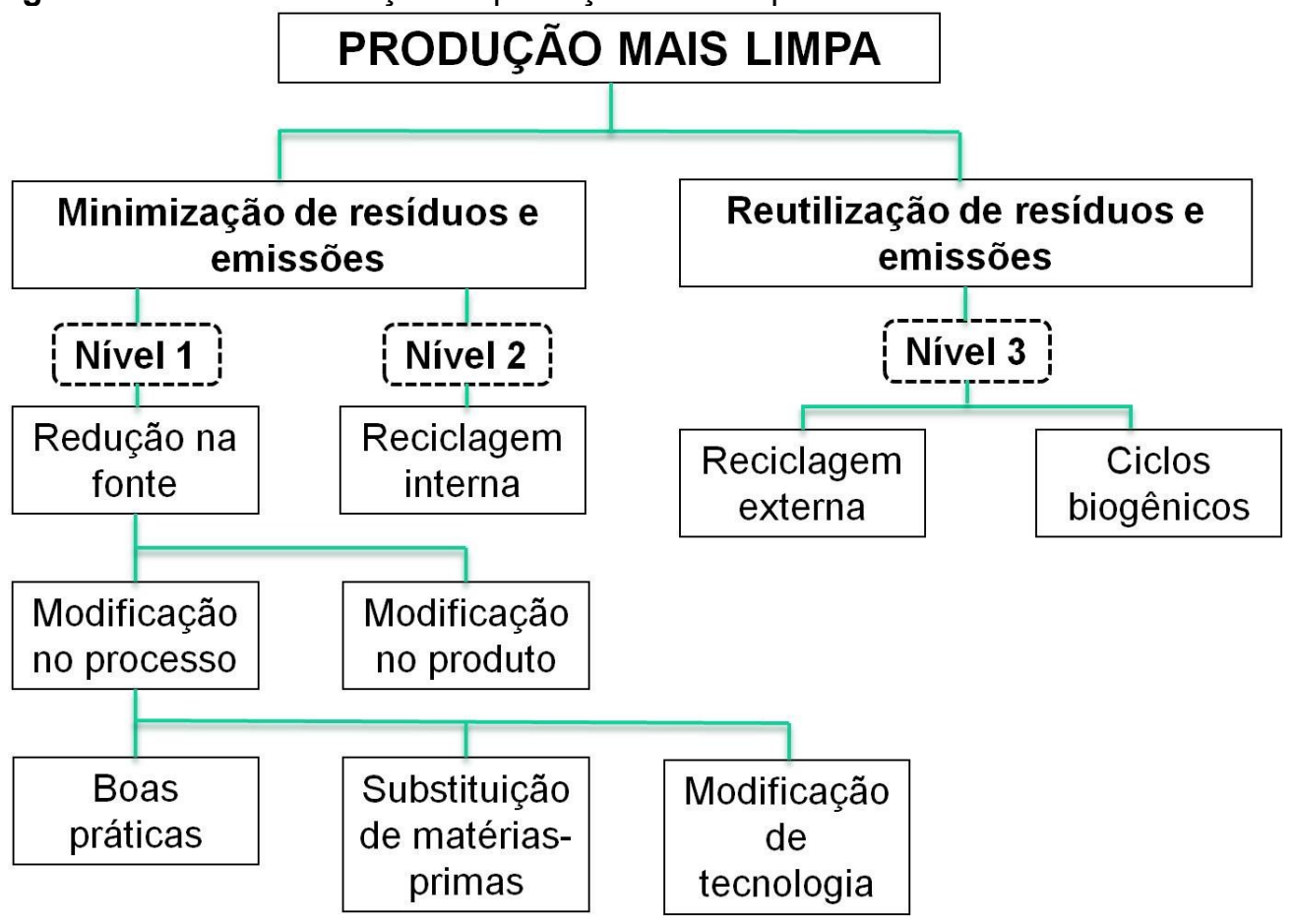

Fonte: UNEP (2002); Medeiros et al. (2007)

A PML, de acordo com Thrane, Nielsen e Christensen (2009), é uma estratégia de prevenção que pode abordar tanto processos de fabricação como produtos, e que interpreta o desenvolvimento de produtos mais limpos como o tipo mais radical de melhoria. Seiffert (2011) afirma que por meio da implantação da PML, é possível a obtenção de soluções que contribuam efetivamente para a resolução de problemas ambientais, uma vez que a metodologia prioriza a identificação de opções de não geração dos resíduos oriundos desses processos produtivos. Ainda, a autora afirma que a produção mais limpa não visa, restritamente, à identificação, quantificação, tratamento e disposição final dos resíduos, mas sim a eliminação da geração dos mesmos.

Complementando, ressalta-se que a PML possui práticas simples e econômicas, uma vez que considera, para fins de análise, apenas a unidade fabril em estudo, ou seja, clientes e fornecedores não englobam o foco de estudo (HINZ; VALENTINA; FRANCO, 2007). Entretanto, Vendrametto et al. (2010) destacam que os fornecedores também possuem papel fundamental na estratégia de PML, uma Revista Produção Online, Florianópolis, SC, v.13, n. 1, p. 233-256, jan./mar. 2013. 
vez que a globalização e a competitividade possuem tendências universais que geram uma reação em cadeia, fazendo com que todas as empresas envolvidas tenham comportamento semelhante em seus processos.

\subsection{A Teoria da Solução Inventiva de Problemas (TRIZ)}

Genrich Altshuler, nascido em 1926 na ex-União Soviética, serviu como consultor na marinha nos anos 1940 para apoiar inventores no processo de patenteamento de invenções. É considerado o criador da teoria de solução inventiva de problemas, genericamente conhecida como TRIZ, originada dos seguintes termos russos: Teorija Rezhenija Izobretatel'skisch Zadach (BACK et al., 2008).

O termo TRIZ é um acrônimo para Teoria da Solução Inventiva de Problemas, que tem sido usado em várias pesquisas (STRATTON; MANN, 2003; LOH; HE; SHEN, 2006; SOO et al., 2006; VINCENT, 2006; ROBLES; NEGNY; LANN, 2008; SCYOC, 2008; DUBOIS; ELTZER; DE GUIO, 2009; LI; HUANG, 2009; MERK; CAVALLUCCI; ROUSSELOT, 2009; ROBLES; NEGNY; LANN, 2009; VERHAEGEN et al., 2009; ZHANG; MAO; ABOURISK, 2009; AHMED et al., 2011; BECATTINI et al., 2011; HOUSSIN; COULIBALY, 2011; KIM et al., 2011; NOVOA et al., 2011; VERHAEGEN et al., 2011; YANG; CHEN, 2011). Esse trabalho, iniciado em 1946, conduziu Altshuler a pesquisar métodos de solução de problemas e identificou que os métodos intuitivos existentes na época não satisfaziam as exigências de invenções da segunda metade do século XX (BACK et al., 2008).

Diversos sistemas diferentes de soluções foram gerados abstraindo princípios inventivos da análise de dados das patentes. E muitas dessas soluções focam as contradições ou conflitos identificados nas soluções inovadoras. Stratton e Mann (2003) e Li e Huang (2009) entendem que a metodologia TRIZ afirma que problemas inventivos podem ser codificados, classificados e resolvidos metodicamente, assim como outros problemas de engenharia.

A abordagem da TRIZ foi amplamente ensinada e difundida na Rússia, porém não emergiu no Ocidente até o final dos anos 80. Carvalho et al. (2011) afirmam que embora sua criação tenha acontecido há diversos anos e vivido grande expansão mundial na década de 2000, a TRIZ ainda não se consolidou no ferramental de desenvolvimento de tecnologias, produtos e serviços de várias empresas brasileiras. Revista Produção Online, Florianópolis, SC, v.13, n. 1, p. 233-256, jan./mar. 2013. 
Os pesquisadores, pioneiramente Altshuler, buscaram por princípios de solução inventiva dos problemas, e para isso, analisaram uma grande quantidade de patentes russas (aproximadamente 2,5 milhões de patentes), sendo pesquisadas, inicialmente, as de design mecânico, observando princípios genéricos e como as soluções patenteadas foram alcançadas (BACK et al., 2008; LI; HUANG, 2009; FRESNER et al., 2010). Assim, conseguiram identificar condições que as teorias de invenções elaboradas deveriam atender, na forma das seguintes leis da evolução para sistemas técnicos:

- Evolução inteligente dos sistemas: os sistemas evoluem em passos discretos;

- Aumentar idealidade: sistemas evoluem em direção à idealidade, caracterizada pelo fornecimento da função técnica, sem causar efeitos nocivos (em termos de esforço, consumo - excessivo - de recursos, etc.);

- Diferente evolução dos elementos do sistema: os elementos do sistema evoluem em diferentes níveis;

- Aumento na dinâmica e controle: os sistemas são dinamizados, o controle sobre a evolução aumenta;

- Aumento da complexidade e posterior diminuição: a complexidade de um sistema aumenta e diminui novamente, depois de atingir certo nível de complexidade;

- Aumento da coordenação: o ritmo dos diferentes elementos de um sistema técnico se torna cada vez mais coordenado;

- Miniaturização: o sistema e seus elementos tendem a se tornar miniaturizados;

- Diminuição na interação humana: a interação humana com o sistema diminui à medida que o mesmo evolui.

Mann (2002) relatou que a TRIZ é uma filosofia, um processo e uma série de ferramentas (Figura 2), e demonstra que a metodologia TRIZ é baseada na fundamentação do conhecimento em design e uma grande quantidade de pesquisas. 
Figura 2 - Visualização hierárquica da TRIZ

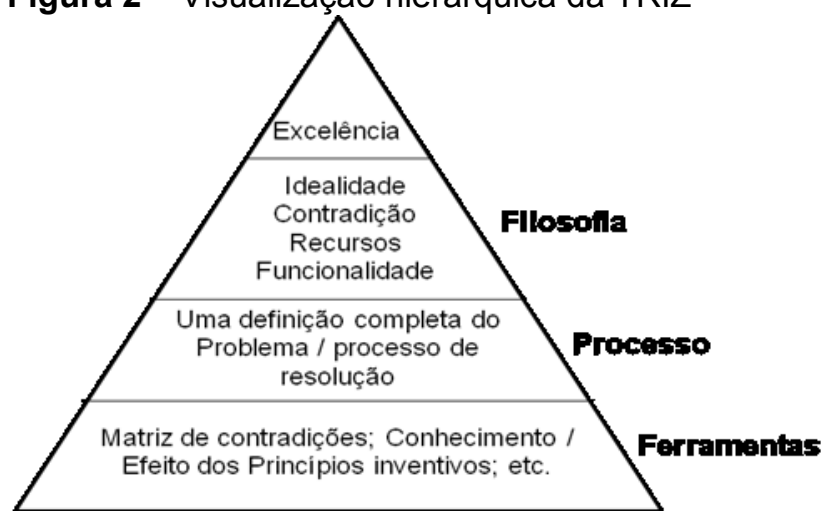

Fonte: Adaptado de Mann (2002)

Além disso, a TRIZ possui diversos conceitos e ferramentas (Figura 3), conforme ilustra Zhang, Mao e Abourisk (2009), provendo abordagens sistemáticas e princípios generalistas para formular e analisar problemas, gerar ideias criativas, e projetar a tendência de evolução de um sistema ou projeto.

Figura 3 - Conceitos e ferramentas da TRIZ

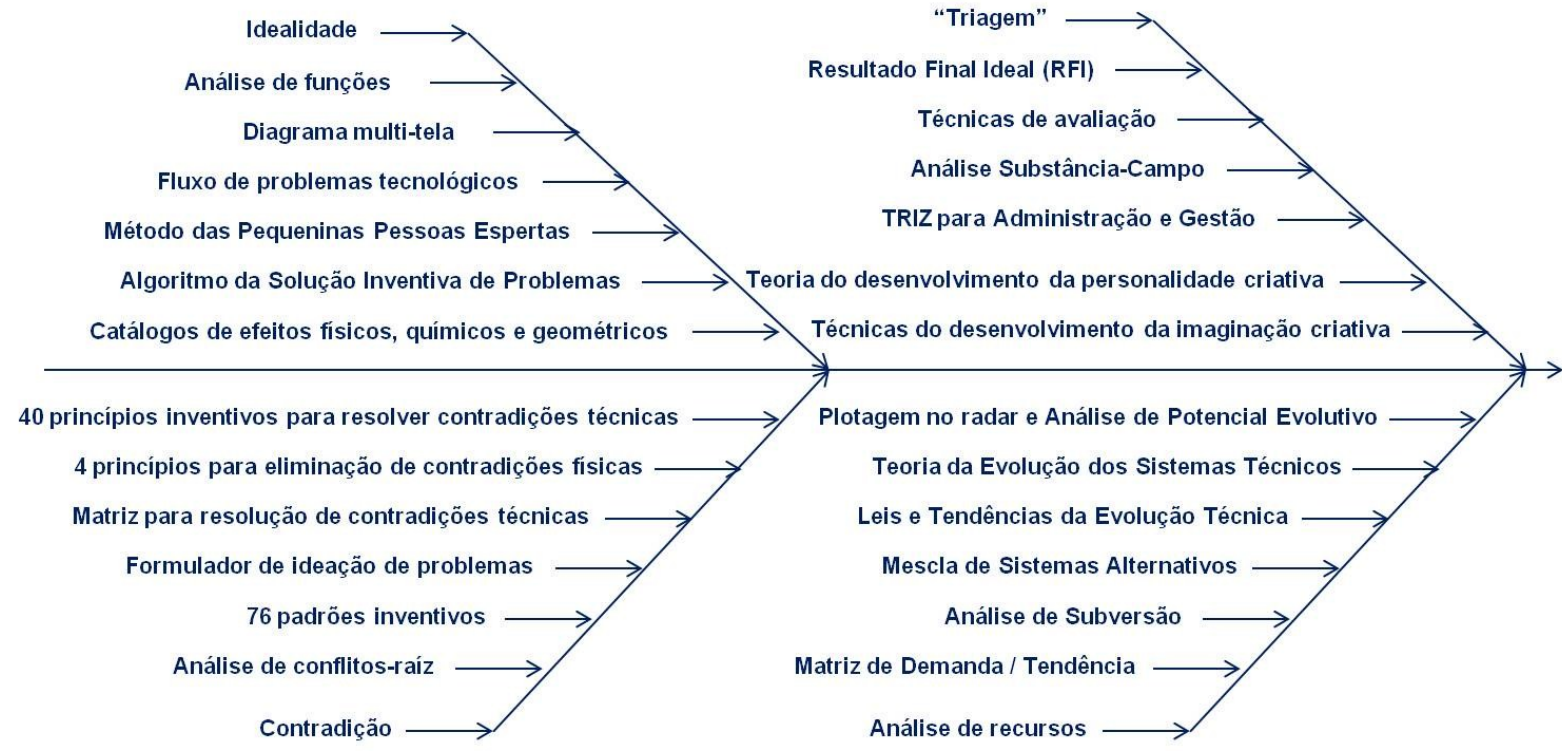

Fonte: Adaptado de Zhang, Mao e Abourisk (2009)

A TRIZ clássica, desenvolvida por Altshuller e seus colaboradores, é composta por métodos para a formulação e a solução de problemas, uma base de conhecimento e leis da evolução para sistemas técnicos - ST's (CARVALHO; BACK, 2001). Carvalho (2007) e Yang e Chen (2011) relatam que, de modo geral, a TRIZ é

Revista Produção Online, Florianópolis, SC, v.13, n. 1, p. 233-256, jan./mar. 2013. 
aplicada da seguinte maneira: um problema inventivo é reformulado em um problema genérico da TRIZ, e então, as ferramentas da metodologia são introduzidas para analisar e propor uma solução geral baseada na mesma (Figura 4). Assim, uma solução genérica é interpretada para resolver um problema inventivo específico. Soares (2008) ressalta que o método se baseia na ideia de que todos os problemas técnicos já foram resolvidos de alguma forma no passado, e os princípios inerentes às suas soluções encontram-se armazenados em bases de dados de patentes.

Figura 4 - Metodologia TRIZ

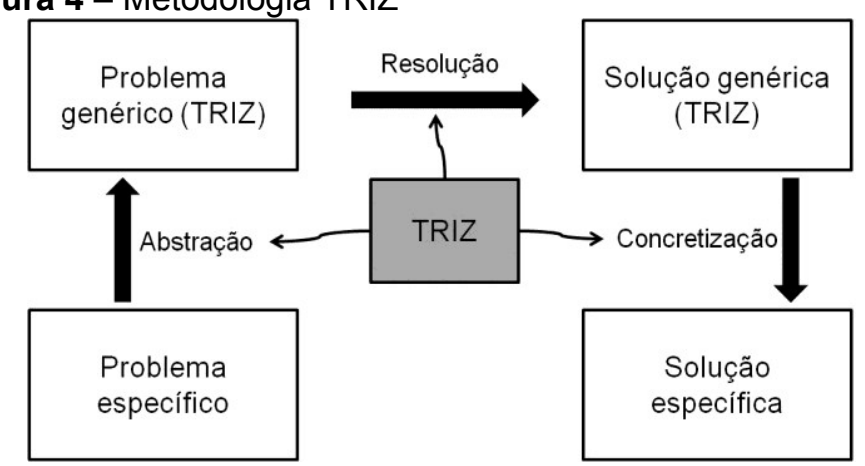

Fonte: Stratton e Mann (2003); Srinivasan e Kraslawski (2006); Carvalho (2007); Li e Huang (2009); Kim et al. (2011); Ahmed et al. (2011); Novoa et al. (2011); Yang e Chen (2011)

Para o entendimento desta metodologia, é necessária, inicialmente, a definição e compreensão clara de seus conceitos fundamentais, os quais são: idealidade, contradições e recursos. Idealidade, segundo Carvalho e Back (2001), é a razão entre o número de funções desejadas e funções indesejadas executadas pelo sistema. O próprio sistema técnico (ST) é entendido, pela TRIZ, como um "preço" pago pela execução de funções desejadas por seus usuários. Portanto, quanto mais evoluído é esse sistema (mais próximo do ideal), menor é o "preço" pago pela sociedade. E é a partir desse conceito que é gerado o resultado final ideal (RFI) que consiste em alcançar a idealidade ou se aproximar ao máximo dela.

Contradições são requisitos conflitantes com relação a um mesmo ST - a melhora de um requisito causa a piora de outro (CARVALHO; BACK, 2001; CARVALHO; HATAKEYAMA, 2003). Carvalho e Back (2001) citam como exemplo uma haste de solda para equipamentos elétricos e eletrônicos, onde a haste não deve ser muito pequena, para não queimar a mão do operador, e nem tão grande, para que o controle sobre o instrumento não seja prejudicado. E os recursos, por Revista Produção Online, Florianópolis, SC, v.13, n. 1, p. 233-256, jan./mar. 2013. 
fim, podem ser definidos como quaisquer elementos do sistema ou dos arredores que ainda não foram utilizados para realizar funções úteis do sistema. Existem casos em que recursos não aproveitados levam a soluções inventivas (CARVALHO; BACK, 2001).

Adicionalmente, Stratton e Mann (2003) e Li e Huang (2009) afirmam que existem três premissas básicas na teoria: a primeira relata que o objetivo é o desenho ideal da solução sem funções nocivas; a segunda premissa afirma que uma solução inventiva envolve a eliminação total ou parcial de uma contradição, e; a terceira diz que o processo inventivo pode ser estruturado.

\section{MÉTODO DA PESQUISA}

Visando atender aos objetivos delineados neste estudo, realizou-se uma pesquisa de caráter qualitativo e natureza descritiva (HAIR Jr. et al., 2006), com estratégia de coleta de dados documental. Trata-se de uma pesquisa teóricoconceitual, conforme classificação de Filippini (1997) e Berto e Nakano (2000), uma vez que foram elaborados conceitos e definições com base na literatura, revisões bibliográficas (GUPTA; VERMA; VICTORINO, 2006; MIGUEL, 2010), bem como experiências dos autores. Assim, foram detectadas convergências e conexões entre os assuntos estudados.

Dentro da base de periódicos da Coordenação de Aperfeiçoamento de Pessoal de Nível Superior (CAPES), foram utilizadas as seguintes palavras-chave para a busca dos trabalhos pesquisados: Teoria da Solução Inventiva de Problemas, produção mais limpa, TRIZ, PML, Theory of Inventive Problem Solving, Cleaner Production e CP.

Foram avaliadas teses e dissertações a respeito do assunto, além de artigos publicados em eventos (nacionais e internacionais) e diversos periódicos: Journal of Cleaner Production, TRIZ Journal, European Symposium on Computer Aided Process Engineering, Computer-aided design, Computer in Industry, Advanced Engineering Informatics, Creativity and Innovation Management, Journal of Loss Prevention in the Process Industries, Reliability Engineering and System Safety, Expert Systems with Applications, Chemical Engineering and Processing, Journal of Materials Processing Technology, Journal of Bionic Engineering, Automation in Revista Produção Online, Florianópolis, SC, v.13, n. 1, p. 233-256, jan./mar. 2013. 
Construction, Procedia Environmental Sciences.

O enquadramento dos trabalhos pesquisados se deu do seguinte modo: procurou-se alinhar cada tese/dissertação/artigo conforme os níveis de atuação da produção mais limpa, com o intuito de se evidenciar qual o maior enfoque de aplicação. Foram consideradas as pesquisas onde foram utilizados conceitos e ferramentas da TRIZ visando à redução de efluentes, resíduos e emissões, minimização de utilização de recursos e uso de insumos sustentáveis, bem como a elaboração de metodologias integradas direcionadas ao ecodesign e projeto de processos, seguindo-se assim as estratégias de PML. Como limitação desta etapa do método, ressalta-se o fato de os trabalhos englobados no nível 1, em modificações no processo, não serem enquadrados dentro de suas três abordagens - boas práticas (housekeeping), substituição de matéria-prima e modificação de tecnologia.

Outras características importantes (objetivos, resultados e considerações finais) das pesquisas também foram estudadas, visando o levantamento de informações acerca dos pontos fortes e fracos, oportunidades e ameaças da integração entre a TRIZ e a PML. Dessa forma, foi possível obter informações para se alcançar os objetivos específicos desta pesquisa.

\section{RESULTADOS E DISCUSSÕES}

\subsection{Relações entre a TRIZ e a PML}

Através das pesquisas realizadas, foi possível constatar que a maioria das aplicações da TRIZ, no que tange a PML, relacionam-se com o ecodesign, seguida pelas melhorias em processos. Ou seja, o enfoque é, essencialmente, em modificações nos produtos (nível 1). A exemplo, Chen e Liu (2001) associaram os 39 parâmetros de engenharia, bem como os 40 princípios inventivos, com os sete elementos da eco-eficiência. O resultado foi a geração de uma nova metodologia para elaboração de produtos eco-inovadores, através de ferramentas da TRIZ, alinhadas a esses sete elementos. Chang e Chen (2003) mostraram exemplos de eco-inovação para cada um dos 40 princípios inventivos, cobrindo uma grande variedade de produtos e processos, considerados - à época - novidades, criativos e 
"eco-amigáveis".

Outra aplicação em produtos da TRIZ foi desenvolvida por Serban et al. (2005), onde foi realizada uma pesquisa a fim de verificar as ferramentas e métodos da TRIZ as quais poderiam ser utilizadas na abordagem do design para o meio ambiente (Design for Environment - DFE). O trabalho buscou incrementar a fase de projeto conceitual, e propôs a referida nova metodologia (alinhando a TRIZ e o DFE) em um estudo de caso. Soares (2008) desenvolveu uma ferramenta criativa de apoio ao desenho de produtos sustentáveis, onde métodos da TRIZ foram incorporados para auxiliar na resolução de conflitos de projeto. Os métodos utilizados foram a análise de contradições e conflitos.

Em outras pesquisas recentes, Yang e Chen (2011) construiram uma metodologia que acelera o design preliminar para a eco-inovação, utilizando-se de estudo baseado em casos (Case-Based Reasoning - CBR) alinhados à TRIZ análise de contradições e leis da evolução. Evidenciou-se, também, que esse não foi o único estudo com sucesso na união entre as duas ferramentas. Trabalhos publicados por Estevez et al. (2006), que integraram os dois referidos métodos para o design inventivo, e Yang e Chen (2009), que buscaram resolver problemas relacionados ao ecodesign e acelerar o padrão evolutivo de um projeto baseado na TRIZ/CBR, também obtiveram resultados efetivos.

Em relação à modificações no processo (nível 1), foram encontradas poucas evidências de aplicações da TRIZ/PML. Li, Rong e Kraslawski (2001) propuseram a utilização integrada da TRIZ com análise termodinâmica, visando a substituição de dois equipamentos no setor de destilação de uma unidade fabril. Os resultados encontrados, através da metodologia, contribuiram para a elaboração de dois fluxogramas detalhados do processo ideal, e a conclusão de que ocorreria redução no consumo de vapor com a troca dos equipamentos em $23,7 \%$ e $27,7 \%$, respectivamente.

Li et al. (2003) utilizaram a análise de conflitos/contradições - derivada da TRIZ - para minimizar as perdas de água em processos industriais, servindo como um eficiente método para tratamento dos conflitos entre os objetivos visados para identificar as alternativas potenciais de prevenção da poluição. Em outro estudo, Li (2004) propôs uma nova metodologia para a síntese conceitual de processos, com 
base na análise de conflitos/contradições, visando a melhoria na tomada de decisão no projeto e síntese, bem como instigar a criatividade nas atividades de projeto.

Ainda em processos, Fresner et al. (2010) buscaram alternativas de eliminar a água de lavagem no processo de decapagem em uma indústria química. Através da chamada "triagem" e da análise de interações (ambas ferramentas da TRIZ), foi possível buscar a solução ideal final para o processo, bem como alternativas de se alcançar a mesma. Inicialmente, foi identificado que o problema se tratava do consumo de água de lavagem utilizada para diluir a película de produtos químicos localizada na superfície das peças da empresa. A partir dessa situação, concluiu-se que a idealidade consistia em uma superfície livre de contaminantes para o próximo processo, sem nenhuma função nociva (perdas de água, desperdício e consumo de energia). Na Tabela 1, encontram-se os principais resultados das pesquisas sobre a TRIZ relacionados aos níveis de atuação da PML.

Outro aspecto observado é quanto ao conceito de idealidade. Conforme apresentado no referencial teórico deste artigo, a idealidade tem como objetivo a ausência ou o mínimo de funções indesejadas no produto/processo/serviço em questão. A PML, simultaneamente, visa a redução de efluentes, resíduos e emissões dentro dos processos produtivos, bem como um consumo racional e sustentável de matéria-prima, além da busca por insumos menos agressivos ao meio ambiente. Assim, pode-se inferir, dentro da perspectiva da integração TRIZ/PML, que a idealidade na PML é a não-geração de efluentes/resíduos/emissões nos produtos, processos produtivos e prestação de serviços, resultando, assim, em situações de inexistência de saídas indesejadas em todos os níveis de atuação da produção mais limpa.

O conceito de contradição também tem relação com a PML. Serban et al. (2005) concluiram, em suas pesquisas, que a TRIZ é considerada uma metodologia promissora, uma vez que muitos projetistas encaram a relação ecodesign/crescimento econômico como uma contradição. Contudo, os mesmos autores, juntamente com Luttropp e Lagerstedt (2006) consideram que essa integração TRIZ/ecodesign auxilia os projetistas a maximizar a utilização dos recursos de um sistema para atingir os objetivos de desenvolvimento de um novo produto com menor custo e sem efeitos indesejados. Portanto, resumidamente, o 
conceito de contradição, dentro da TRIZ/PML pode ser compreendido como produzir em maior quantidade e com melhor qualidade, utilizando-se de menor quantidade de entradas (inputs) e gerando menos saídas (outputs).

Por fim, o conceito de recursos também possui alinhamento com as estratégias de produção mais limpa, principalmente no que se refere àqueles que o sistema não utiliza dentro de suas funções. Na literatura, há uma quantidade significativa de estudos que detectaram recursos os quais, após serem incorporados aos sistemas técnicos, trouxeram benefícios técnicos, ambientais e econômicos aos mesmos. Não foram encontrados exemplos com uso explícito da TRIZ/PML com esse enfoque, entretanto, estudos de Coutinho, Rodrigues e Silva (2009) e Silva et al. (2009), podem ser enquadrados nesta categoria.

Tabela 1 - Resultados da pesquisa quanto à abordagem dos artigos (TRIZ/PML)

\begin{tabular}{|c|c|c|}
\hline RIZ aplicada à proc & mais limpa (F & ) - Abordagem dos autores pesquis \\
\hline \multirow[t]{2}{*}{$\begin{array}{l}\text { Nível } 1 \text { (Redução na } \\
\text { fonte) }\end{array}$} & $\begin{array}{l}\text { Modificações no } \\
\text { produto }\end{array}$ & $\begin{array}{l}\text { Chen e Liu (2001), Braunschweig e Irons } \\
\text { (2002), Chang e Chen (2003), Serban et al. } \\
\text { (2005), Estevez et al. (2006), Kobayashi (2006), } \\
\text { Soares (2008), Yang e Chen (2009), Sheng e } \\
\text { Soo (2010), Bandera, Filippi e Motyl (2011), } \\
\text { Yang e Chen (2011a), Yang e Chen (2011b) } \\
\text { Russo, Regazzoni e Montecchi (2011), Russo } \\
\text { et al. (2011). }\end{array}$ \\
\hline & $\begin{array}{l}\text { Modificações no } \\
\text { processo }\end{array}$ & $\begin{array}{l}\text { Li, Rong e Kraslawski (2001), Li et al. (2003), } \\
\text { Stratton e Mann (2003), Li (2004), Srinivasan e } \\
\text { Kraslawski (2006), Nicoletti e Quinello (2009), } \\
\text { Fresner et al. (2010), Kim et al. (2011). }\end{array}$ \\
\hline Nível 2 & $\begin{array}{l}\text { Reciclagem } \\
\text { interna }\end{array}$ & $\begin{array}{l}\text { Coutinho, Rodrigues e Silva (2009), Silva et al. } \\
\qquad(2009) .\end{array}$ \\
\hline Nível 3 & $\begin{array}{l}\text { Reciclagem } \\
\text { externa e ciclos } \\
\text { biogênicos }\end{array}$ & Não foram encontradas evidências. \\
\hline
\end{tabular}

Fonte: Elaborado pelos autores

\subsection{Discussões: pontos fortes, pontos fracos, oportunidades e ameaças na integração TRIZ/PML}

O fato de ser amplamente utilizada por empresas de grande porte, tendo em vista a inovação, bem como a estruturação organizada de todo esse processo, torna 
a metodologia bastante eficiente e eficaz na geração de novos produtos e processos.

A TRIZ pode oferecer, na análise funcional, todas as funções desejadas e indesejadas do produto ou processo. Com isso, facilita-se a priorização e resolução dos problemas encontrados, podendo essa ação ser executada sistematicamente, de acordo com os interesses e preferências da organização. No caso de se utilizar o enfoque da produção mais limpa, é possível direcionar os esforços na eliminação das funções indesejadas as quais possuem impacto ambiental negativo mais prejudicial no produto/processo/serviço em estudo. No exemplo de Fresner et al. (2010), isso está claro quando os autores realizaram a análise de funções, conforme relatado anteriormente (item 4.1) e ilustrado na Figura 5.

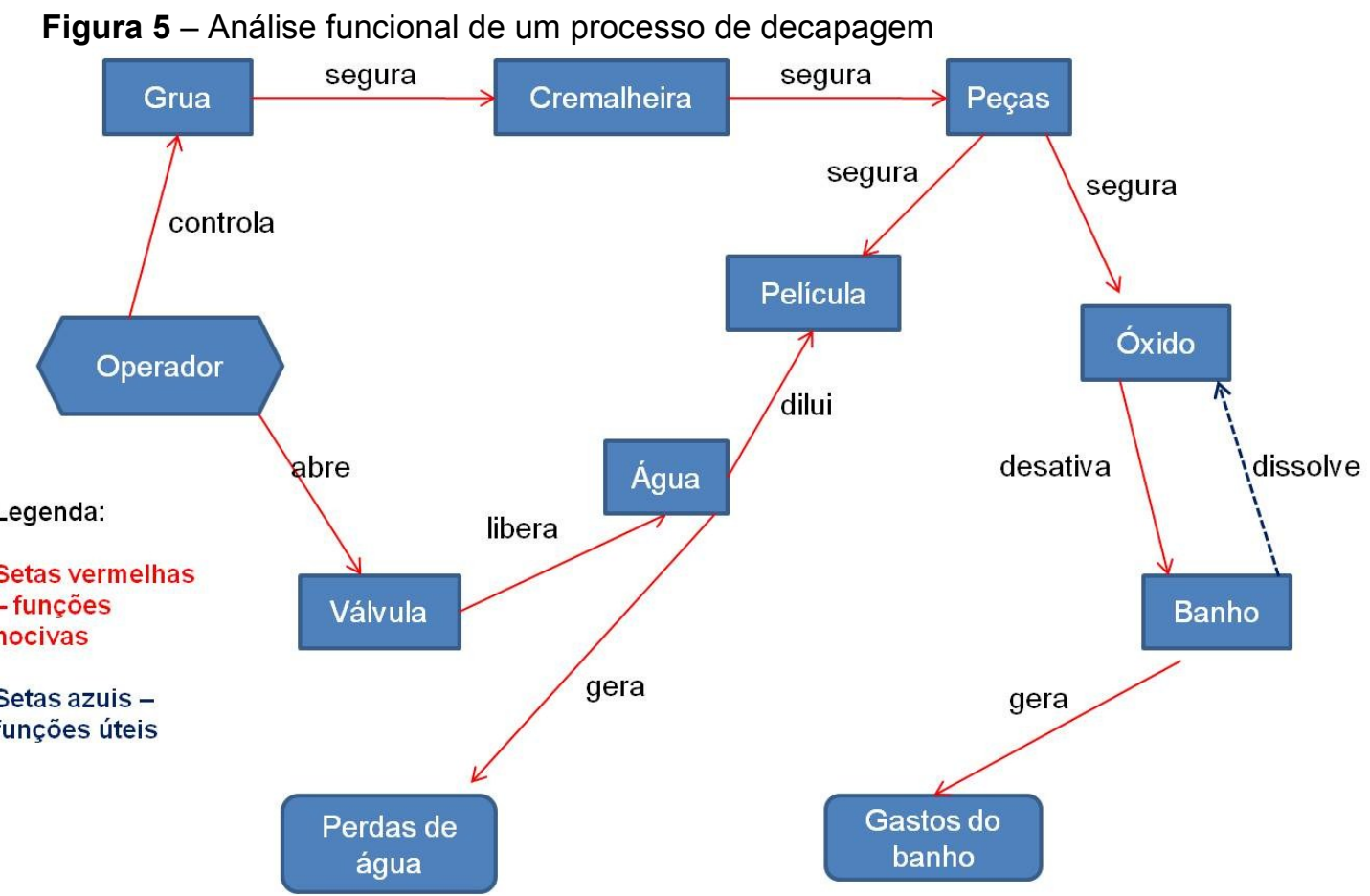

Fonte: Fresner et al. (2010)

Outra característica importante da TRIZ é o fato de ser uma metodologia flexível, uma vez que, em alguns casos, essa é modificada para se adaptar a cada ramo específico de atuação (ex: alimentos, agroindústria, farmacêutica, produção, etc.). O que é um benefício significativo, pois em diversos setores da indústria há a necessidade de melhorias ambientais.

Revista Produção Online, Florianópolis, SC, v.13, n. 1, p. 233-256, jan./mar. 2013. 
No entanto, a principal virtude encontrada (e mais observada durante a pesquisa) é a fácil integração da TRIZ com o ecodesign, união essa que crescentemente tem sido aplicada, com o objetivo de gerar produtos menos agressivos ao meio ambiente, alinhando isso a um custo menor de fabricação. A aplicação dos 40 princípios inventivos de Altshuller, seja com a análise de contradições ou não, fornece uma importante base de conhecimentos para que as organizações tenham condições de desenvolver projetos de produtos/processos sustentáveis.

Em relação aos pontos fracos, foi observado que no nível 2 (reciclagem interna) da PML não foram encontradas evidências do uso explícito da TRIZ. Entretanto, alguns trabalhos, indiretamente, utilizaram-se de conceitos básicos como o de recursos, por exemplo. É o caso dos estudos de Coutinho, Rodrigues e Silva (2009) e Silva et al. (2009). Não se obteve relatos da aplicação da metodologia no que se refere ao nível 3 da PML, portanto, referências de soluções inventivas relacionadas à reciclagem externa e ciclos biogênicos não foram disponibilizadas.

As aplicações de sucesso para o design de produtos e processos não estão disponíveis em publicações na literatura aberta. Consequentemente torna-se mais difícil, inclusive, a maior disseminação e aplicação da ferramenta, uma vez que não há dados disponíveis a respeito de melhorias realizadas, aumento no lucro na minimização de desperdícios, etc. (SRINIVASAN; KRASLAWSKI, 2006). Entretanto, em todos os trabalhos pesquisados, ocorreram contribuições à organização em estudo, desde a implantação de alternativas para melhoria até o levantamento de potenciais soluções, uma vez que nem todos os trabalhos envolveram a aplicação de fato - das ideias propostas, mas contribuíram para a tomada de decisão na melhoria da organização. Isso ressalta o quanto a metodologia TRIZ fomenta a criatividade e instiga a busca de alternativas para o melhoramento dos produtos/processos/serviços, mesmo em equipes com pouco conhecimento técnico.

As poucas aplicações em processos se devem à dificuldade de adaptar os princípios inventivos, pois os mesmos foram elaborados, essencialmente, com base em melhorias e/ou projeto de produtos. Apesar disso, foram encontrados trabalhos realizados nessa área: Fresner et al. (2010), Srinivasan e Kraslawski (2006), Li et al. (2003) e Stratton e Mann (2003) são alguns dos exemplos. Salienta-se aqui que, 
sem uma análise de funções detalhada, a metodologia será bastante prejudicada, uma vez que o objetivo é buscar contradições e elementos nocivos que possam ser eliminados - ou reduzidos - do processo, sendo para isso necessário o maior conhecimento possível acerca do processo a ser estudado.

Há, também, a oportunidade de se estudar exemplos de princípios inventivos específicos para a PML, uma vez que a mesma possui características peculiares, assim como, por exemplo, a indústria química. Isso se reforça com base no estudo de Grierson et al. (2003), que iniciou a discussão de como os 40 princípios inventivos de Altshuler podem ser aplicados diretamente aos problemas químicos. Outra oportunidade levantada é quanto à fase de mapeamento e elaboração de fluxogramas de processo, onde se sabe que, em algumas situações, podem ocorrer limitações de obtenção de dados e informações, limitando as pesquisas. A análise funcional é uma alternativa, pois essa ferramenta permite o estudo aprofundado de uma etapa do processo a ser incrementado.

Srinivasan e Kraslawski (2006) afirmam que a metodologia TRIZ possui limitações no que diz respeito ao projeto de processos, devido ao seu caráter abstrato e consequentes dificuldades de aplicação, em especial no que se refere ao uso da matriz de contradições. A complexidade dessa etapa, onde a resolução de uma contradição pode - provavelmente - gerar outras contradições, pode tornar esse processo interminável para a resolução de determinado problema (SRINIVASAN; KRASLAWSKI, 2006).

Outra possível ameaça é a variação dos resultados da aplicação da metodologia, ou seja, não se tem uma previsibilidade de resultados. Nicoletti e Quinello (2009) justificam isso relatando que o grau de vivência, conhecimento e experiência das pessoas envolvidas pode variar, sendo esses fatores decisivos no sucesso ou fracasso da TRIZ. Porém, não se pode confundir isso com engessamento criativo, pois a criação de soluções inventivas consiste, necessariamente, em mapear novas alternativas (NICOLETTI; QUINELLO, 2009). Assim, as pessoas menos experientes na equipe também contribuem, uma vez que o risco de sofrer com o engessamento criativo é menor. Nesse caso, faz-se necessário um equilíbrio na força de trabalho da organização, no que se refere à experiência, conhecimento e capacidade perceptiva. 
Para melhor ilustrar os resultados obtidos nesta pesquisa, a Figura 6 retrata, de forma sucinta, na forma de matriz SWOT (Strenghts - Forças, Weaknesses Fraquezas, Opportunities - Oportunidades e Threats - Ameaças), as evidências encontradas a respeito da utilização da TRIZ em melhorias relacionadas à PML.

Figura 6 - Evidências acerca de aplicações da TRIZ na PML

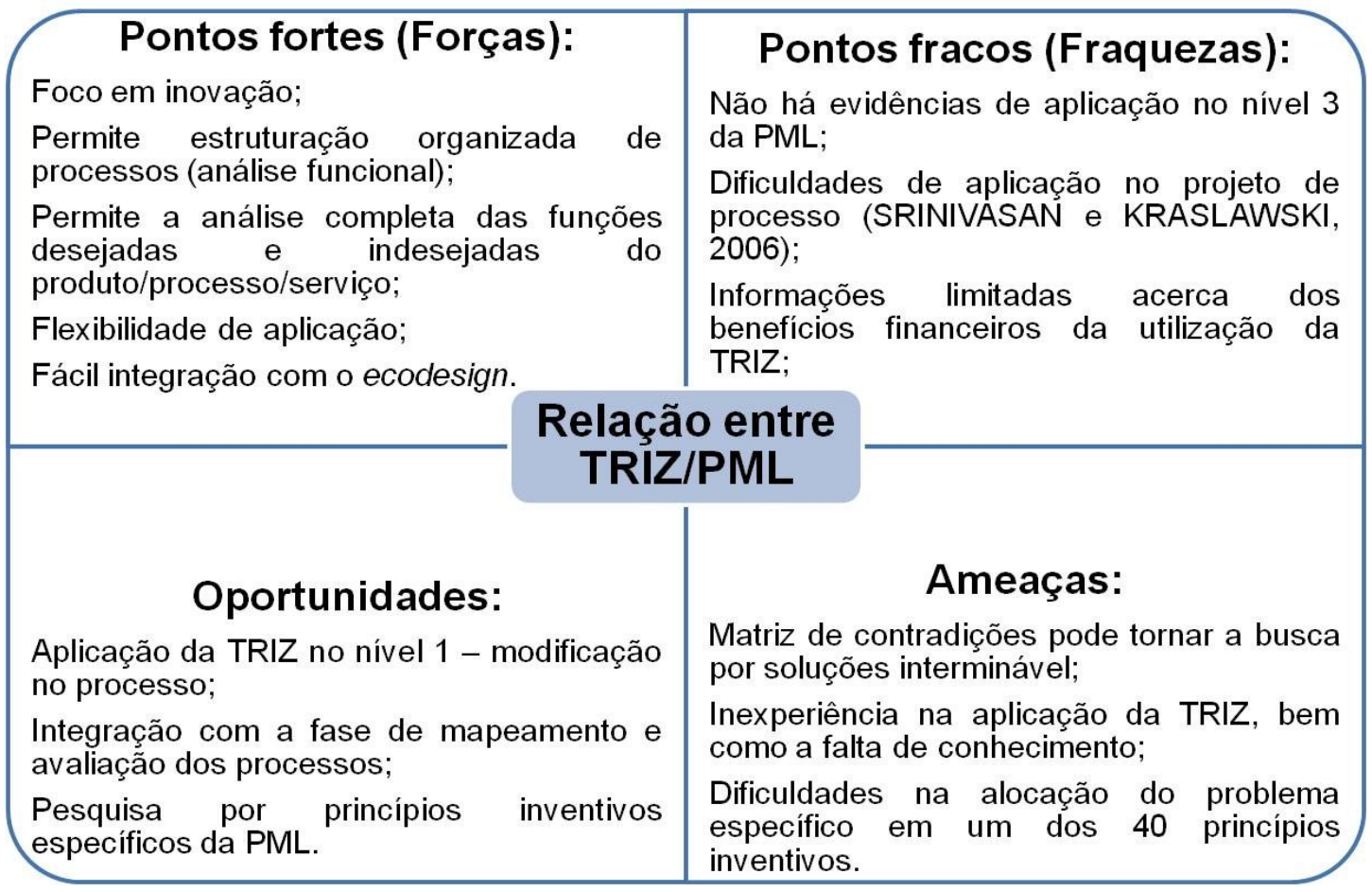

Fonte: elaborado pelos autores

\section{CONSIDERAÇÕES FINAIS E OPORTUNIDADES FUTURAS}

Este trabalho teve como objetivo principal analisar e discutir, por meio da literatura, as possibilidades de utilização da TRIZ como metodologia de suporte à produção mais limpa (PML), visando à geração de soluções criativas nessa área, uma vez que há crescente necessidade da melhoria contínua e sustentável nos produtos e processos das organizações.

De modo geral, pode-se observar que as organizações que se utilizaram da TRIZ obtiveram potenciais benefícios na busca de soluções inventivas e sustentáveis, em especial no que se refere ao nível 1 da PML, nas modificações de produto (ecodesign), foco da maioria dos trabalhos encontrados na literatura. Ainda no nível 1, mas em processos, foram encontradas menos evidências da utilização da 
TRIZ. E dentro dessas publicações, uma quantia significativa foi direcionada ao aumento da segurança nos processos, reduzindo os riscos químicos e emissões nocivas. As publicações pesquisadas trouxeram benefícios no que diz respeito à análise dos processos, bem como da busca por melhorias nos mesmos.

Conforme relatado anteriormente, ao utilizar a matriz de contradições, há de se ter o cuidado para que o processo de resolução de problemas não se torne interminável, o que pode ocorrer devido ao elevado poder de abstração que esta ferramenta da TRIZ possui.

Através da TRIZ, os projetistas têm a oportunidade de definir a melhor direção para melhorar os produtos. A integração da metodologia no ecodesign ajuda os designers a maximizar a utilização dos recursos de um sistema para atingir os objetivos de desenvolvimento de um novo produto com menor custo e sem efeitos indesejados (SERBAN et al., 2005). Fresner et al. (2010) ainda ressaltam que, a metodologia baseada na TRIZ/PML proporcionou uma nova interpretação das estratégias de produção mais limpa, além de ser uma ferramenta valiosa para coordenar equipes que busquem alternativas de PML, podendo-se envolver pessoas com pouco embasamento de engenharia. Observou-se também que os conceitos fundamentais (idealidade, contradição e recursos) da TRIZ têm potencial para contribuir para as estratégias de redução e/ou reutilização de efluentes, resíduos e emissões dos processos produtivos estudados, gerando, consequentemente, benefícios econômicos.

Por fim, constatou-se que a metodologia TRIZ tem potencial para ser mais explorada no desenvolvimento e melhoria de processos, uma vez que a análise funcional, juntamente com a análise de recursos, os conceitos fundamentais de contradição, idealidade e posterior elaboração do resultado final ideal (RFI), permitem compreender a situação estudada de maneira detalhada, bem como esclarece todas as funções desejadas e indesejadas ocorrentes no processo em estudo, definindo as oportunidades e limitações do ambiente em questão. Desse modo, a integração TRIZ/PML pode servir como direcionadora na busca da otimização dos processos produtivos, promovendo a redução do impacto ambiental negativo dos mesmos, bem como prover a melhoria contínua focada em inovação, convergindo com os conceitos da estratégia da produção mais limpa.

\section{REFERÊNCIAS}

AHMED, Rizwan, et al. Design of safety-critical systems using the complementarities of success and failure domains with a case study. Reliability Engineering and

System Safety, v. 96, n. 1, p. 201-209, 2011.

Revista Produção Online, Florianópolis, SC, v.13, n. 1, p. 233-256, jan./mar. 2013. 
ALTSHULLER, Genrich Saulovich. Erfinden - wege zur lösung technischer Probleme. PI Verlag, 1998.

BACK, Nelson, et al. Projeto integrado de produtos: planejamento, concepção e montagem. Florianópolis: Manole, 2008.

BANDERA, Camillo; FILIPPI, Stefano; MOTYL, Barbara. A survey on systematic innovation strategies for product design and development. In: INTERNATIONAL CONFERENCE ON INNOVATIVE METHODS IN PRODUCT DESIGN, 2011, Venice, Italy. Proceedings... Venice, 2011. Disponível em:

http://www.improve2011.it/Full Paper/104.pdf. Acesso em: 26 ago. 2011.

BECATTINI, Niccolò; BORGIANNI, Yuri; CASCINI, Gaetano; ROTINI, Federico. Model and algorithm for computer-aided inventive problem analysis. Computeraided design, 2011. Disponível em:

<http://www.sciencedirect.com/science/article/pii/S0010448511000583>. Acesso em: 22 mar. 2011.

BERKEL, René Van. Cleaner Production perspectives 1: CP and industrial development. UNEP - industry and environment, v. 24, n. 1-2, p. 28-32, 2001.

BERTO, Rosa Maria Villares de Souza; NAKANO, Davi Noboru. A produção científica nos anais do Encontro Nacional de Engenharia de Produção: um levantamento de métodos e tipos de pesquisa. Produção, v. 9, n. 2, p. 65-76, 2000.

CARVALHO, Marco Aurélio de. Metodologia IDEATRIZ para a ideação de novos produtos. 2007. 254f. Tese (Doutorado em Engenharia de Produção) Universidade Federal de Santa Catarina, Florianópolis, 2007.

CARVALHO, Marco Aurélio de; BACK, Nelson. Uso dos conceitos fundamentais da TRIZ e do método dos princípios inventivos no desenvolvimento de produtos. $3^{\circ}$ CONGRESSO BRASILEIRO DE GESTÃO DE DESENVOLVIMENTO DE

PRODUTO, 3, 2001, Florianópolis. Anais eletrônicos... Florianópolis: UFSC, 2001. Disponível em: http://www.decarvalho.eng.br/macartigoiiicbgdp.pdf. Acesso em: 10 de dezembro, 2010.

CARVALHO, Marco Aurélio de, et al. Criatividade em Problemas de Projeto e Processo com Auxílio da TRIZ (Teoria da Solução Inventiva de Problemas) / Inovação Sistemática. CONGRESSO BRASILEIRO DE GESTÃO DE DESENVOLVIMENTO DE PRODUTO, 8,. 2011, Porto Alegre. Anais... Porto Alegre: UFRGS, 2011.

CARVALHO, Marco Aurélio de; HATAKEYAMA, Kazuo. Solução inventiva de problemas e engenharia automotiva: a abordagem da TRIZ. 2003. Disponível em: $<$ http://www.aditivaconsultoria.com/artigoengautomotivaeaerospacialmarcoekazuo.pdf>. Acesso em: 27 de fevereiro, 2011. 
CHAI, Kah-Hin; ZHANG, Jun; TAN, Kay-Chuan. A TRIZ based method for new service design. Journal of Service Research, v. 8, n. 1, p. 48-66, 2005.

CHANG, Hsiang-Tang; CHEN, Jahau Lewis. Eco-innovative examples for 40 TRIZ Inventive Principles. TRIZ Journal, v. 8, 2003.

CHEHEBE, José Ribamar. Análise do ciclo de vida de produtos: ferramenta gerencial da ISO 14.000. Rio de Janeiro: Qualitymark, CNI, 1997.

CHEN, Jahau Lewis; LIU, Chih-Chen. An eco-innovative design approach incorporating the TRIZ method without contradiction analysis. The Journal of Sustainable Product Design, v. 1, n. 4, p. 263-272, 2001.

COUTINHO, Janclei Pereira; RODRIGUES, Luciano Brito; SILVA, Cristiano Alves da. Implantação de uma estratégia para reaproveitamento do óleo de fritura residual em um restaurante industrial. In: SIMPÓSIO DE ENGENHARIA DE PRODUÇÃO, 16, . 2009, Bauru. Anais eletrônicos... Bauru: Unesp, 2009. Disponível em: http://www.simpep.feb.unesp.br/anais simpep.php?e=4. Acesso em: 24 set. 2011.

DUBOIS, Sébastien; ELTZER, Thomas; DE GUIO, Roland. A dialectical based model coherent with inventive and optimization problems. Computers in Industry, v. 60, n. 8 , p. 575-583, 2009.

ESTEVEZ, Isabel, et al. Le raisonnement à partir de cas est-il utilisable pour l'aide à la conception inventive. 14 $^{\circ}$ atelier de Raisonnement à Partir de Cas, n. 30-31, p. 123-129, 2006.

FILIPPINI, Roberto. Operations management research: some reflections on evolution, models and empirical studies in OM. International Journal of Operations and Production Management, v. 17, n. 7, p. 655-670, 1997.

FRESNER, Johannes; JANTSCHGI, Jürgen; BIRKEL, Stefan; BÄRNTHALER, Josef; KRENN, Christina. The theory of inventive problem solving (TRIZ) as option generation tool within cleaner production projects. Journal of Cleaner Production, v. 18, n. 2 , p. 128-136, 2010.

GRIERSON, Billy, et al. 40 Principles - Chemical Illustrations. TRIZ Journal, v. 7, 2003.

GUPTA, Sushil; VERMA, Rohit; VICTORINO, Liana. Empirical research published in production and operations management (1992-2005): trends and future research directions. Production and Operations Management, v. 15, n. 3, p. 432-448, 2006.

HAIR, Joseph F. P. J., et al. Fundamentos de métodos de pesquisa em administração. São Paulo: Bookman, 2006.

HINZ, Roberta Tomasi Pires; VALENTINA, Luiz V. Dalla; FRANCO, Ana Claudia. Monitorando o desempenho ambiental das organizações através da produção mais 
limpa ou pela avaliação do ciclo de vida. Revista Produção Online, v. 7, n. 3, nov. 2007. Disponível em:

<http://www.producaoonline.org.br/index.php/rpo/article/view/66/66> .

HOUSSIN, Rémy; COULIBALY, Amadou. An approach to solve contradiction problems for the safety integration in innovative design process. Computers in Industry, v. 62, n. 4, p. 398-406, 2011.

KIM, Junghwan, et al. Application of TRIZ creativity approach to chemical process safety. Journal of Loss Prevention in the Process Industries, v. 22, n. 6, p. 10391043, 2011.

KUBOTA, Flávio Issao; ROSA, Leandro Cantorski da. A TRIZ (Theory of Inventive Problem Solving) aplicada à produção mais limpa: uma abordagem preliminary. ENCONTRO NACIONAL DE ENGENHARIA DE PRODUÇÃO, 31., 2011, Belo Horizonte. Anais... Belo Horizonte: UNIFEI, 2011.

KUBOTA, Flávio Issao; SILVA FILHO, Daves Pereira; ROSA, Leandro Cantorski da. Produção mais limpa: introdução de práticas no melhoramento de processos em usina escola de laticínios. SIMPÓSIO DE ENGENHARIA DE PRODUÇÃO, 17., 2010, Bauru. Anais eletrônicos... Bauru: Unesp, 2010. Disponível em: http://www.simpep.feb.unesp.br/anais simpep.php?e=5. Acesso em: 22 nov. 2010.

$\mathrm{LI}$, Xiaoning. Conflict-based method for conceptual process synthesis. $104 \mathrm{f}$. Tese (Doutorado em Ciência - Tecnologia) - Lappeenranta University of Technology, Laapeenranta, 2004.

LI, Te-Sheng; HUANG, Hsing-Huang. Applying TRIZ and Fuzzy AHP to develop innovative design for automated manufacturing systems. Expert Systems with Applications, v. 36, n. 4, p. 8302-8312, 2009.

LI, Xiao-Ning; RONG, Ben-Guang; KRASLAWSKI, Andrzej. TRIZ-based creative retrofitting of complex distillation processes - an industrial case study. European Symposium on Computer Aided Process Engineering, v. 11, p. 439-444, 2001.

LOH, Han Tong; HE, Cong; SHEN, Lixiang. Automatic classification of patent documents for TRIZ users. World Patent Information, v. 28, n. 1, p. 6-13, 2006. LUTTROPP, Conrad; LAGERSTEDT, Jessica. EcoDesign and the ten golden rules: generic advice for merging environmental aspects into product development. Journal of Cleaner Production, v. 14, n. 15-16, p. 1396-1408, 2006.

MANN, Darrel. Hands-on Systematic Innovation. leper: CREAX, 2002.

MEDEIROS, Denise Dumke de, et al. Aplicação da produção mais Limpa em uma empresa como ferramenta de melhoria contínua. Produção, v. 17, n. 1, p. 109-128, 2007. 
MERK, Cecilia Zanni; CAVALLUCCI, Denis; ROUSSELOT, François. An ontological basis for computer aided innovation. Computers in Industry, v. 60, n. 8, p. 563-574, 2009.

MIGUEL, Paulo Augusto Cauchick. Metodologia de pesquisa em engenharia de produção e gestão de operações. Rio de Janeiro: Elsevier, 2010.

NICOLETTI, José Roberto; QUINELLO, Robson. Aplicação da metodologia TRIZ para análise e reengenharia do sistema de controle ambiental aplicado em uma instalação agro-industrial no Estado de São Paulo. In: SIMPÓSIO DE ADMINISTRAÇÃO DA PRODUÇÃO, LOGÍSTICA E OPERAÇÕES INTERNACIONAIS, 12,. São Paulo. Anais eletrônicos... São Paulo: FGV-EAESP, 2009. Disponível em:

<http://www.simpoi.fgvsp.br/arquivo/2009/artigos/E2009 T00214 PCN19741.pdf>. Acesso em: 29 jun. 2011.

NOVOA, Roberto Duran, et al. Inventive problem solving based on dialectical negation, using evolutionary algorithms and TRIZ heuristics. Computer in Industry, v. 62 , n. 4 , p. 437-445, 2011.

PIMENTA, Handson Cláudio Dias; GOUVINHAS, Reidson Pereira. Implementação da produção mais limpa na indústria de panificação de Natal-RN. In: ENCONTRO NACIONAL DE ENGENHARIA DE PRODUÇÃO, 27, . 2007. Anais... Foz do Iguaçu: ABEPRO, 2007. Associação Brasileira de Engenharia de Produção.

RIVERA, Alejandro; GONZÁLEZ, Jorge Silvio; CARRILLO, Raúl; MARTíNEZ, José María. Operational change as a profitable cleaner production tool for a brewery. Journal of Cleaner Production, v. 17, n. 2, p. 137-142, 2009.

ROBLES, Guillermo Cortes; NEGNY, Stéphane; LANN, Jean Marc Le. Design acceleration in chemical engineering. Chemical Engineering and Processing, $v$. 47, n. 11, p. 2019-2028, 2008.

ROBLES, Guillermo Cortes; NEGNY, Stéphane; LANN, Jean Marc Le. Case-based reasoning and triz: a coupling for innovative conception in chemical engineering.

Chemical Engineering and Processing: Process Intensification, v. 48, n. 1, p. 239249, 2009.

RUSSO, Davide; REGAZZONI, Daniele; MONTECCHI, Tiziano. Eco-design with TRIZ laws of evolution. Procedia Engineering, v. 9, p. 311-322, 2011.

SCYOC, Karl Van. Process safety improvement - quality and target zero. Journal of Hazardous Materials, v. 159, n. 1, p. 42-48, 2008.

SEIFFERT, Maria Elizabete Bernardini. Gestão ambiental: instrumentos, esferas de ação e educação ambiental. 2 ed. São Paulo: Atlas, 2011. 
SERBAN, Daniela, et al. A TRIZ approach to design for environment. Kluwer Academic Journal, 2005.

SHENG, Isaac Lim Sing; SOO, Teoh Kok. Eco-efficient product design using theory of inventive problem solving (TRIZ) principles. American Journal of Applied Sciences, v. 7, n. 6, p. 852-858, 2010.

SILVA, Monick Alexandre da, et al. Gerenciamento dos resíduos como ferramenta para uma produção mais limpa no ramo de panificação. In: SIMPÓSIO DE ENGENHARIA DE PRODUÇÃO, 16,. Bauru. Anais eletrônicos... Bauru: Unesp, 2009. Disponível em: <http://www.simpep.feb.unesp.br/anais simpep.php?e=4>. Acesso em: 30 set. 2011.

SOARES, Marina Arminda Ribeiro. Biomimetismo e ecodesign: desenvolvimento de uma ferramenta criativa de apoio ao design de produtos sustentáveis. $84 \mathrm{f}$. Dissertação (Mestrado em Engenharia do Ambiente) - Universidade Nova de Lisboa, Lisboa, 2008.

SOO, Von-Wun, et al. A cooperative multi-agent platform for invention based on patent document analysis and ontology. Expert Systems with Applications, v. 31, n. 4, p. 766-775, 2006.

SRINIVASAN, Robert; KRASLAWSKI, Andrzej. Application of the TRIZ creativity enhancement approach to design of inherently safer chemical processes. Chemical Engineering and Processing, v. 45, n. 6, p. 507-514, 2006.

STRATTON, Robert; MANN, Darrell. Systematic innovation and the underlying principles behind TRIZ and TOC. Journal of Materials Processing Technology, v. 139, n. 1-3, p. 120-126, 2003.

THRANE, Mikkel; NIELSEN, Eskild Holm; CHRISTENSEN, Per. Cleaner production in danish fish processing: experiences, status and possible future strategies. Journal of Cleaner Production, v. 17, n. 3, p. 380-390, 2009.

UNITED NATIONS ENVIRONMENTAL PROGRAMME (UNEP). Changing production patterns - learning from the experience of national cleaner production centres. Paris: United Nations Environment Programme (UNEP). Division of Technology, Industry, and Economics (DTIE), 2002.

VENDRAMETTO, Oduvaldo; PALMERI, Nivaldo; NETO, Geraldo Cardoso O; PERRETI, Osvaldo D'Angelo. Cleaner production: a growing movement in brazilian companies. Revista Produção Online, v.10, n.1, p. 49-70, mar. Disponível em: $<$ http://www.producaoonline.org.br/index.php/rpo/article/view/240>.

VERHAEGEN, Paul-Armand, et al. Relating properties and functions from patents to TRIZ trends. CIRP Journal of Manufacturing Science and Technology, v. 1, n. 3, p. $126-130,2009$. 
VERHAEGEN, Paul-Armand, et al. Identifying candidates for design-by-analogy. Computers in Industry, v. 62, n. 4, p. 446-459, 2011.

VINCENT, Julian F. V. The materials revolution. Journal of Bionic Engineering, v. 3, n. 4, p. 217-234, 2006.

WINKLESS, Barry; MANN, Darrel. Food product development and the 40 inventive principles. TRIZ Journal, v. 5, 2001.

YANG, Cheng Jung; CHEN, Jahau Lewis. TRIZ method for eco-innovation with casebased reasoning. INTERNATIONAL SYMPOSIUM ON ENVIRONMENTALLY CONSCIOUS DESIGN AND INVERSE MANUFACTURING, 6,. 2009.

Proceedings..., 2009.

YANG, Cheng Jung; CHEN, Jahau Lewis. Accelerating preliminary eco-innovation design for products that integrates case-based reasoning and TRIZ method. Journal of Cleaner Production, v. 19, n. 9-10, p. 998-1006, 2011a.

YANG, Cheng Jung; CHEN, Jahau Lewis. Forecasting the design of eco-products by integrating TRIZ evolution patterns with CBR and Simple LCA methods. Expert Systems with Applications, 2011b.

ZHANG, Jing; SHANG, Jie. Research on developing environmental protection industry based on TRIZ theory. Procedia Environmental Sciences, v. 2, p. 13261334, 2010.

ZHANG, X.; MAO, X.; ABOURIZK, S. M. Developing a knowledge management system for improved value engineering practices in the construction industry. Automation in Construction, v. 18, n. 6, p. 777-789, 2009.

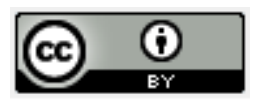

Artigo recebido em 26/10/2011 e aceito para publicação em 24/01/2012. 